\title{
Efficiency of Using the Diffie-Hellman Key in Cryptography for Internet Security ${ }^{*}$
}

\author{
Ghaith Alomari ${ }^{a}$ and Anas Aljarah ${ }^{b}$ \\ $\mathbf{A}_{\text {chicago state university, department of mathematics and computer science, chicago/united states }}$ \\ (ORCID: 0000-0002-5196-7049)

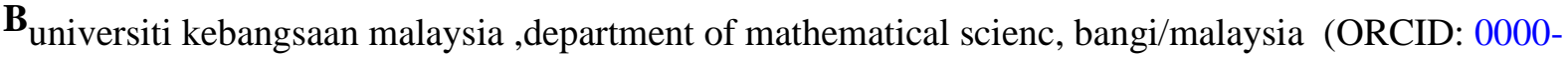 \\ 0002-9033-6928)
}

Article History: Received: 10 November 2020; Revised 12 January 2021 Accepted: 27 January 2021; Published online: 5 April 2021

\begin{abstract}
The businesses generate an "intranet" to hang about connected to the internet but secured from possible threats. Data integrity is quite a issue in security and to preserve that integrity we tends to develop as to provides the better encryption processes for security. In this work we will make a encryption harder with enhanced public key encryption protocol for the security and we will talk about the applications for proposed work. We will enhance the hardness in security by humanizing the Diffie-Hellman encryption algorithm by making changes or adding some more security codes in up to date algorithm. Network security has become more important to not public computer users, organizations, and the military. With the start of the internet, security became a major disquiet and the history of security allows a better understanding of the emergence of security technology. The internet structure itself allowed for many security threats to occur.When the architecture of the internet is modified it can decrease the possible attacks that can be sent across the network. Knowing the attack methods, allows for the suitable security to appear. By means of the firewalls and encryption mechanisms many businesses protected themselves from the internet.The firms crank out an "internet" to hold around connected into this world wide web but procured from potential dangers. Data ethics is a significant dilemma in protection and also to conserve integrity we all are inclined to grow concerning furnishes exactly the encryption procedures such as the security. Inside this job we'll earn a encryption tougher using improved general security protocol to your own stability and we're going to discuss the software for projected work. We'll improve the hardness of stability by humanizing that the Diffie Hellman encryption algorithm by generating alterations or including a few far more stability codes up to date algorithm. Network safety has gotten more very important to perhaps not people users, associations, and also the army. With all the beginning of internet, stability turned into a significant vexation along with the foundation of safety makes it possible for a superior comprehension of the development of technology. Even the online arrangement itself enabled for most security dangers that occurs. After the structure of this world wide web is altered it could diminish the probable strikes which may be transmitted from the other side of the community. Recognizing the assault procedures, permits the acceptable stability to arise. With this firewalls and security mechanics many companies shielded themselves out of the world wide web.
\end{abstract}

Keywords: Diffie-Hellman, Private Key, Public Key, Cipher, Security

\section{Introduction}

Communicating is your main portion in just about any sort of system in which makes it feasible to move data in 1 node into some other. Communication demands security and quality to get far better performance as well as also for approval of both client and users' businesses. Quality depends upon size and also various additional facets of system nevertheless protection is incredibly anxiety parameter in system because it's independent of system dimensions and sophistication. Stability has gotten more crucial to computer consumers, associations, and also the army. Security turned into a big concern with all the introduction of online and also the foundation of safety makes it possible for a superior mastery of the development of technology. Even the online arrangement itself enabled for all security dangers that occurs. The changed structure of this net can decrease the probable strikes which might be transmitted from the other side of the community. Recognizing the assault procedures, permits 
the proper stability to arise. Data ethics is a significant dilemma in stability and also to keep up integrity we are inclined to boost to furnishes exactly the encryption procedures such as stability. Inside our planned job we offer more rapid encryption using improved general key encryption protocol for both stability and suggested job might be put into place in to almost any media to supply much better safety. We've improved the hardness from stability by accentuating the Diffie Hellman encryption algorithm with the addition of a few far more stability codes from algorithm. (Council, 2000)

Even the full area of community safety is huge and within a evolutionary point. The variety of review encircles a concise heritage dating back to into net's infancy and also the present advancement in system safety. The desktop understanding of this net, its own vulnerabilities, assault techniques via the web, and stability technological innovation is equally crucial and hence they're assessed so as to comprehend the investigation currently being conducted now.

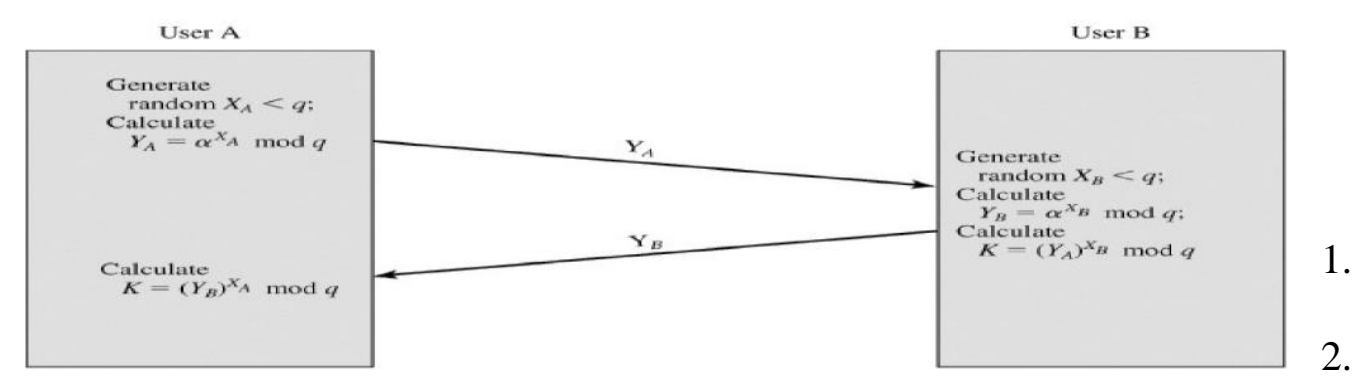

\subsection{Diffie-Hellman Key Exchange Technique}

Step 1: Global Public Components: Prime number $q ; \alpha<q$ along with $\alpha$ is a crude origin of $q$.

Step-2: Consumer an Essential Era: Consumer B Crucial Era:

Step3: Why Pick out personal XA XA $<q$

Select personal $X B X B$

Calculate People $Y B Y B=\alpha X B \bmod q$

Step5: Calculation of Magic Formula Crucial by Consumer A:

Tired of Solution Critical by Consumer B:

The End Result is the two sides possess Traded a magic formula price. What's more, mainly because XA along with XB are confidential, an adversary just includes got the next substances to do the job together: $q$, a, YA, also $Y B$. Ergo, the adversary is made to choose a different logarithm to decide on the main element. For Instance, to Decide on the personal key of consumer, a adversary Need to calculate. (Sethi, 2015)

$X B$

The adversary may subsequently Figure out the Essential $K$ At an identical fashion as consumer calculates it. The safety of this Diffie Hellman key market is within the simple fact, whilst it's not too difficult to estimate exponentials modulo q prime, and it's quite tricky to determine different logarithms. For big windmills, the latter action would be deemed infeasible.

Figure) reveals that a Easy protocol which produces The use of this Diffie Hellman calculation and market. Guess person A wants to establish a experience of user and use a key to encrypt messages on this relationship. Consumer 
A may crank out a onetime confidential secret XA, compute YA, also ship it into your own consumer B. User B reacts by producing a individual worth $\mathrm{XB}$, calculating $\mathrm{YB}$, also delivering $\mathrm{YB}$ to consumer $\mathrm{A}$. each users are now able to figure out the crucial.

The public worth $\mathrm{q}$ and also $\alpha$ would have to become understood beforehand. Instead, user could select values for $\mathrm{q}$ along with $\alpha$ and comprise these from the initial message. The protocol portrayed in Figure inch is insecure contrary to a Man in the Middle strike. The Essential market Routine is exposed to this kind of attack as it doesn't authenticate the Participants. To conquer the vulnerability Using electronic signatures and Public key certifications can be effective.

\section{2- Literature review}

Farshid Farhat et al. has centered upon diagnosis, authentication and key agreement routine of UMTS systems using security style installation has several flaws within the instance of shared freshness of vital arrangement, DoS-attack immunity, and also successful bandwidth intake. Inside this newspaper that they believe UMTS AKA plus other suggested approaches. They then make clear the flaws of this prior frameworks indicated for its UMTS AKA protocol. (Farhat, 2011)

Emmanuel Bresson et al. has researched that the Group Diffie Hellman protocols for authenticated key exchange (AKE) are intended to extend a pool of gamers having a shared key that might later on be properly used, as an instance, to attain multicast content ethics. Through time, numerous schemes are supplied. (Bresson, 2016)

F. Lynn Mcnulty has attracted attention towards this societal and national perspective of the part of encryption is going to be certainly one of those identifying topics because of our civilization at the nineteenth century. Encryption is mentioned from Michael Baum, chairman of this Information Security Committee of the American Bar Association, as "an empowering technologies that gives employers, their business associates, clients and endusers with all the capacities to bring the required info demanded and assistance exactly what they desire just as far faster speed and also much more rigorously and securely". Ubiquitous electronic communications are going to bring about a protected atmosphere to run personal events and digital trade and also perhaps a Kafkaesque earth laid bare by electronic fingerprints suggesting our just about every trade and notions. (Harn, 2014)

SANS Institute data Sec reading-room has researched that the summary of this Diffie Hellman essential trade algorithm and also examine a few shared cryptographic methods being used around the online now that feature Diffie Hellman. The solitude conditions for consumers typically clarified inside the conventional newspaper record entire world are expected in world wide web trades now. Safe and secure electronic communications are quite crucial portion for online e commerce, falsified privacy for professional medical info, etc. In easy case, safe and secure relationships amongst distinct parties that are communication across the Web are currently a need. It's a wonderful and omnipresent algorithm utilized in most protected connectivity methods online. In a age as soon as the life of "older" technological innovation can on occasion be measured in weeks, this algorithm is presently observing its 25 th anniversary whenever it's playing with an active part in Internet protocols that are important. DH can be a technique for tracking a shared key between two parties, even in real time, and within an untrusted network. A common secret will be crucial between 2 functions who might perhaps not need ever hauled formerly, therefore they are able to reestablish their messages. All these protocols will need to get talked about in quick in regard to the specialized utilization of this $\mathrm{DH}$ algorithm and also the condition of the protocol expectations demonstrated or being specified.

\section{3.. Analyzation of problem}

Just as Encryption turned into a critical device for averting the dangers to info tool and sharing to carry on the information integrity consequently, we'll be emphasizing protection boosting by boosting the hardness of security process from various system. For mandatory search we're taking care of renowned encryption algorithm "Diffie Hellman". We're suggesting that the Improvement for Diffie Hellman Algorithm subsequent to analyzing the topics of Diffie Hellman. Network safety has gotten more crucial to computer users, both associations, and also the army. Using the debut of the net, stability turned into a significant worry and also the foundation of safety makes it possible for a superior comprehension of the development of technology. Even the online arrangement itself enabled for most security dangers that occurs. The structure of this world wide web, if modified might lower 
the feasible strikes which might be transmitted from the other side of the community. Recognizing the assault procedures, permits the proper stability to arise. Many companies shield themselves out of your internet with firewalls and security mechanics. The firms produce an "internet" to stay on the world wide web but procured from potential dangers. Data ethics is a significant dilemma in stability and also to keep up integrity we are inclined to boost to furnishes exactly the encryption procedures such as stability. For mandatory search we're taking care of renowned encryption algorithm "Diffie Hellman". Inside our planned job we'll create encryption tougher using improved general key encryption protocol for both securities also certainly will talk about the software for projected work. We'll improve the hardness of stability by accentuating the Diffie Hellman encryption algorithm by simply generating alterations or including a few far more stability codes from algorithm.

The security system We're employing is straightforward (such as Since you'll find a number of people key algorithm nevertheless within this specific particular encryption procedure, a few farther refinements are potential because it's maybe not difficult coded. After a few further changes that this algorithm turned into than just other people secret algorithm as following calculating personal secret $\mathrm{K}$ we are able to employ a mathematical system to generate our personal secret secure. however, it's impossible in RSA and DSA as they're hardcoded.

Diffie Hellman crucial trade, also known as Exponential key trade, can be really a system of computing which uses amounts increased to certain abilities to create decryption keys about the grounds of parts which will never be completely transmitted, so which makes the job of an prospective code-breaker mathematically overpowering. To execute Diffie Hellman, the 2 ending users Alice and Bob, even though conveying within a station that they understand to become confidential, mutually acknowledge favorable full numbers $\mathrm{p}$ and $\mathrm{q}$, for example $\mathrm{p}$ is a quality number and that's actually a generator of de. The generator is lots which, if increased to favorable whole number forces less-than de, not produces precisely the exact same effect for almost any two these kinds of whole amounts. The worth of $\mathrm{p}$ could possibly be huge however, also the worthiness of $\mathrm{q}$ is commonly tiny.

After Alice and Bob have consented de and q Privately, they choose optimistic whole number individual keys that $\mathrm{a}$ and $\mathrm{b}$, so less than the prime-number modulus $\mathrm{p}$. Neither consumer divulges their private secret to anybody; they collect those amounts or produce them down or save them anyplace. Subsequent, Alice and Bob calculate people secrets that $\mathrm{a} \mathrm{b}$ and $* *$ predicated in their own keys in line with this formulation.

\section{4-Mechanism}

To achieve the set objectives, we will do the research in following steps:

Measure Inch: deep analysis of the safety Flaws in system is going to be finished and security prospective will undoubtedly be obtained into consideration.

Measure Two: Investigation of Diffie Hellman Algorithm to feel the safety in vital exchange procedure is going to be finished.

Measure 3: Examine and suggested Algorithm With improvement comes to contour inform of algorithm.

Measure 4: Right after Establishing of suggested Algorithm, execution of suggested algorithm is going to be analyzed on do compiler using $\mathrm{c}$ all language.

Measure 5: Encryption Is Going to Be displayed together with Demonstration in kind of encoded info along with Decrypted Information.

Proposed technique

Sender Ending

Measure 1 ): $\mathrm{Xa}<\mathrm{q}$ (Person may Pick some Random variety significantly less than $\mathrm{q}$ )

Measure Two: $\mathrm{Ya}=\mathrm{a}^{\wedge} \mathrm{Xa} \bmod (\mathrm{Ya}$ Is Really a General secret of sender) 
General secret of recipient Timing is now an individual secret)

Measure 6: Encrypt each correspondence of Simple Text utilizing pow

Receiver Ending

Measure 1): $\mathrm{Xb}<\mathrm{q}$ (Person may Choose some Random variety significantly less than $\mathrm{q}$ )

Of recipient)

Measure 3: $\mathrm{K}-\mathrm{P}=\mathrm{Ya}^{\wedge} \mathrm{Xb}$ mod $\mathrm{q}$ (in which Ya will be A public secret of sender is a individual secret)

Measure 6: Decrypt each single letter of cipher Text utilizing pow

In Which,

One) $q$ is a quality quantity.

Two) a can be a origin of primary variety q.

Result

Assessment Parameters

one) Entropy

Two) Histogram

Resultant Keys

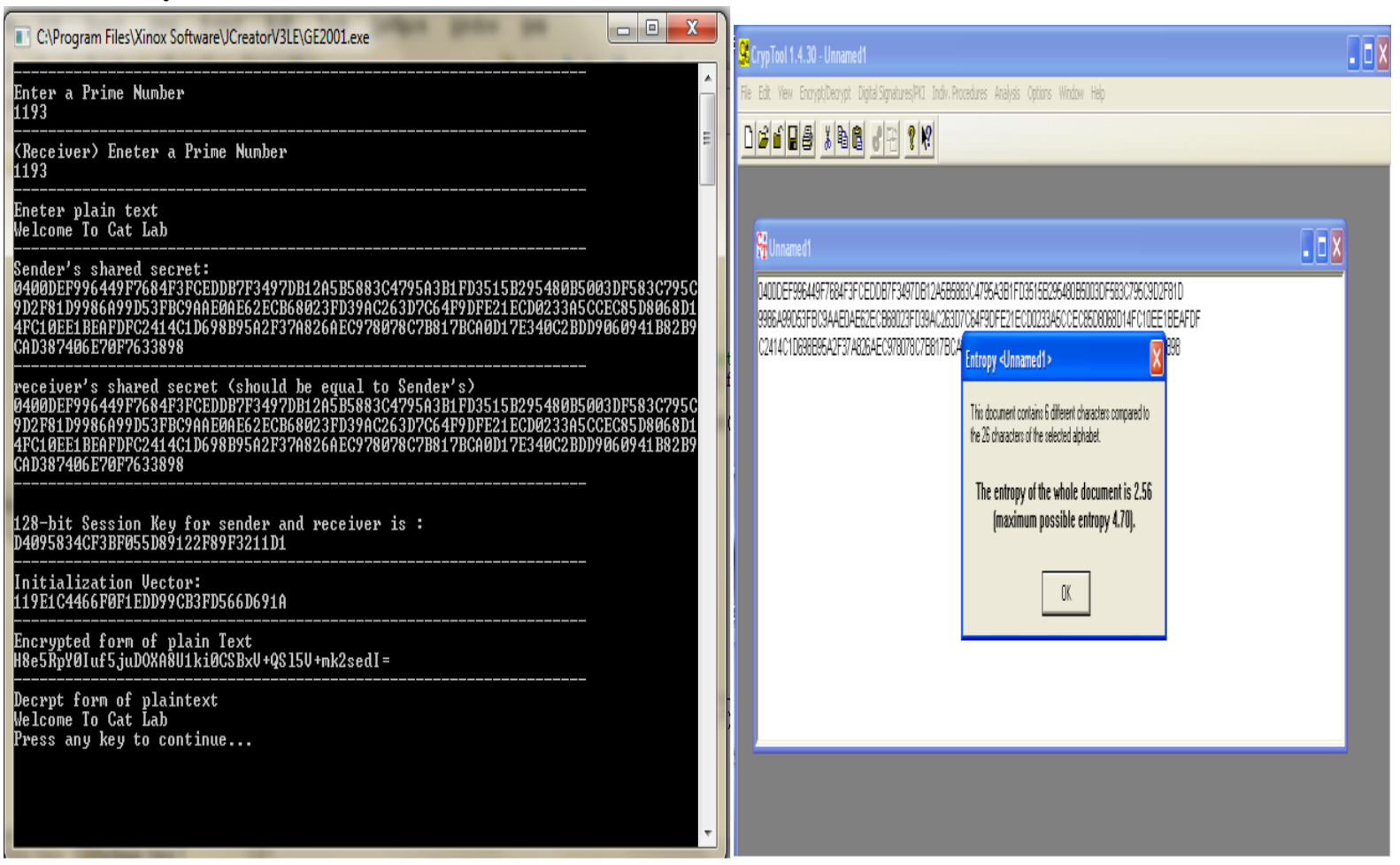




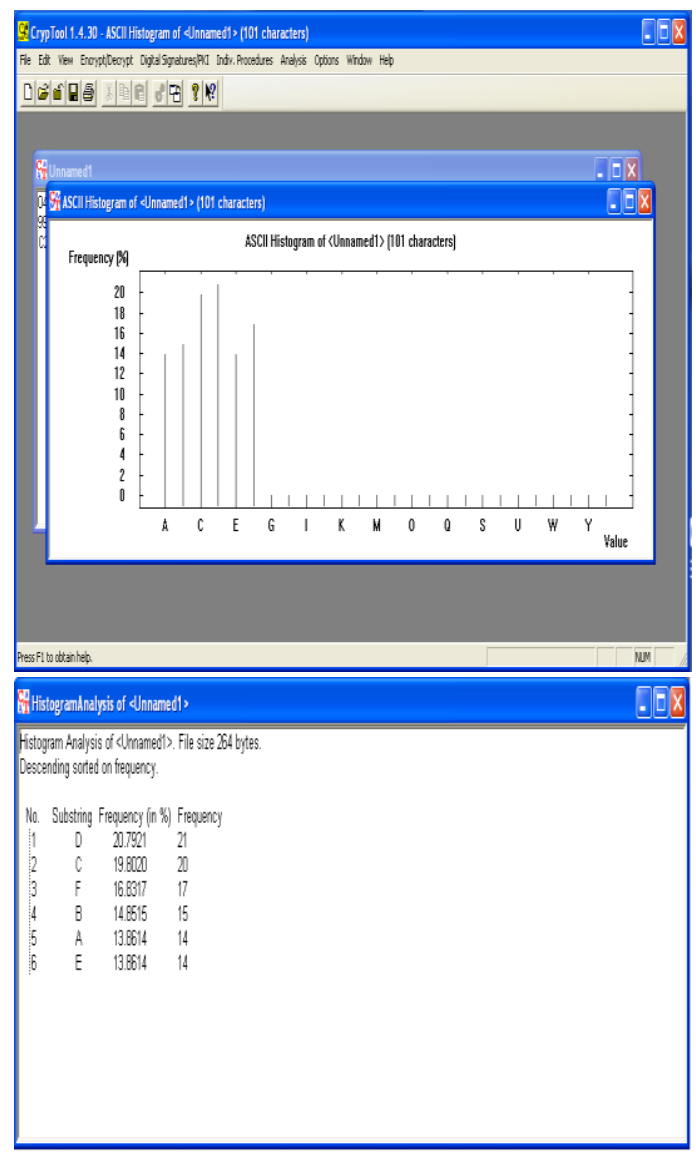

\section{Conclusion}

The Intent of This Research would be to Deliver some Remedy to greater Encryption calculations and attempt to supply much better security for e-mail and To other internet services. The Encryption and Decryption applications accessible all time. The Diffie Hellman key Exchange algorithm has proved to become perhaps one of one of the absolute most interesting essential Supply approaches being used now. But You Have to be alert of this Information that though the algorithm remains protected from passive eavesdropping, It's perhaps not of necessity guarded from busy strikes Because of this particular rationale, the Diffie Hellman algorithm Ought to Be complemented having the authentication mechanism. This strategy for crucial supply Seems to be just one of those Favorite processes utilized in clinic now.

\section{References}

[1]. Council, N. R., (2000). Communicating is your main portion in just about any sort of system in which makes it feasible to move data in 1 node into some other. Communication demands security and quality to get far better performance as well as also for approval of both client and, s.l.: https://www.ncbi.nlm.nih.gov/books/NBK44714/.

[2]. Sethi, P., (2015). The End Result is the two sides possess Traded a magic formula price. What's more, mainly because $X A$ along with $X B$ are confidential, an adversary just includes got the next substances to do the job together: $q, a$, YA, also YB. Ergo, the adversary is made, s.l.: https://www.hindawi.com/journals/jece/2017/9324035/.

[3]. Farhat, F., (2011). Farshid Farhat et al. has centered upon diagnosis, authentication, and key agreement routine of UMTS systems using security style installation has several flaws within the instance of shared freshness of vital arrangement, DoS-attack immunity, and also suc, s.l.: https://eprint.iacr.org/2011/045.

[4]. Bresson, E., (2016). Emmanuel Bresson et al. has researched that the Group Diffie Hellman protocols for authenticated key exchange (AKE) are intended to extend a pool of gamers having a shared key that might later on be properly used, as an instance, to attain multicast conte, s.l.: https://www.iacr.org/archive/asiacrypt2001/22480292.pdf.

[5]. Harn, L., (2014). F. Lynn Mcnulty has attracted attention towards this societal and national perspective of the part of encryption is going to be certainly one of those identifying topics because of our civilization at the nineteenth century. Encryption is mentioned from M, s.l.: http://h.web.umkc.edu/harnl/papers/J8\%202014.pdf. 